\title{
Educación Deportiva en segundo de educación primaria. Percepciones del alumnado y el profesorado respecto a una experiencia de co-enseñanza
}

\section{Sport Education in second-grade primary education. Students and teachers' perceptions of a co-teaching experience}

FEDERICo Puente-MAXERA

Facultad de Formación del Profesorado y Educación. Universidad de Oviedo. España

Campus de Llamaquique. C/ Aniceto Sela, s/n. 33005-Oviedo

fedepuentem@hotmail.com

ORCID: https://orcid.org/0000-0003-3579-1116

\section{ANTONIO MÉNDEZ-GIMÉNEZ}

Facultad de Formación del Profesorado y Educación. Universidad de Oviedo. España

Campus de Llamaquique. C/ Aniceto Sela, s/n. 33005-Oviedo

mendezantonio@uniovi.es

ORCID: https://orcid.org/0000-0003-0078-7053

\section{DiEGO MARTÍNEZ-DE-OJEDA}

Consejería de Educación de la Región de Murcia. España

C/ Río Guadiana, s/n. 30331-Lobosillo, Murcia.

dieigomop@yahoo.es

Recibido: 02-03-2019. Aceptado: 15-09-2019.

Cómo citar / Citation: Puente-Maxera, F., Méndez-Giménez, A., y Martínez de Ojeda, D. (2019). Educación Deportiva en segundo de educación primaria. Percepciones del alumnado y el profesorado respecto a una experiencia de co-enseñanza, Ágora para la Educación Física y el Deporte, 21, 74-95.

DOI: https://doi.org/10.24197/aefd.0.2019.74-95

Resumen: Partiendo de los principios de la Educación Deportiva, el presente estudio se ha propuesto: (a) examinar el impacto de una experiencia de co-enseñanza en segundo curso de educación primaria sobre la competencia, la cultura deportiva y el entusiasmo del alumnado, así como (b) conocer las percepciones del profesorado y alumnado respecto a la intervención. De forma específica, se indagaron posibles diferencias según el género, el país de procedencia y el grupo-clase de pertenencia. La muestra estuvo formada por dos maestros con desigual experiencia respecto al modelo (uno experto y otro novel) y 34 estudiantes ( 22 chicos y 12 
chicas), con edades comprendidas entre los 7 y 8 años $(M=7.53)$, pertenecientes a un colegio ubicado en el sureste de España. Se llevó a cabo una unidad de 12 sesiones sobre un predeporte de balonmano denominado "los cinco pases". La información fue recogida mediante un cuestionario (alumnado), diarios (docentes) y entrevistas (alumnado y profesorado). Los resultados muestran mejoras significativas en cada una de las metas que persigue el modelo. Se advierte especial incidencia de los aspectos que definen al modelo, entre los que destacan las fases inherentes a la temporada o la asunción de roles. Los hallazgos parecen confirmar las bondades que se derivan de conjugar estrategias de co-enseñanza con la Educación Deportiva y extrapolan sus efectos a alumnado de cursos iniciales de educación primaria.

Palabras clave. Educación Física; educación deportiva; co-enseñanza; educación primaria

Abstract: This study has two main goals: (a) to examine the impact of a Sport Education coteaching experience on second-grade students' competence, sport literacy and enthusiasm; and (b) to know teachers and students' perceptions of the intervention. Additionally, there were explored differences according to gender, pupils' country of origin and classroom group. The sample was composed by two teachers with unequal teaching-model experience (expert and novel) and 34 students ( 22 boys and 12 girls) ranging in age from 7 to 8 years old $(M=7.53$ ), from a state school in southeastern Spain. A 12-sessions season of a handball-based pre-sport game called "five passes" was carried out. Information was collected through a questionnaire (students), diary notes (teachers) and interviews (both students and teachers). Results showed significant improvements on model's primary goals (competence, literacy and enthusiasm). It was also revealed a positive impact from some of the model's benchmarks (e. g., season phases or roles and responsibilities). Most relevant findings seem to confirm the benefits from mixing co-teaching strategies with Sport Education, while they suggest its extrapolation to early primary education settings.

Keywords: Physical Education; sport education; co-teaching; primary education.

\section{INTRODUCCIÓN}

Asumir una educación inclusiva y de calidad supone brindar oportunidades al alumnado para un aprendizaje holístico (Santos, 2000). Entre las numerosas iniciativas didácticas que han surgido con dicho propósito, la co-enseñanza ha ocupado un espacio relevante (Conderman, Bresnahan y Pedersen, 2008). El término co-enseñanza o docencia compartida alude a la estrategia didáctica que implica a “dos o más docentes que trabajan de manera colaborativa con el fin de impartir docencia a un grupo heterogéneo de estudiantes en un espacio común” (Friend y Cook, 2007, p.113). Tradicionalmente, la co-enseñanza se ha centrado en la interacción entre un docente generalista y otro de apoyo (p. e., educador especial). Así, una amplia producción ha puesto el foco sobre contextos inclusivos, con alumnado de necesidades educativas 
especiales, tanto en enseñanza general (Keefe y Moore, 2004) como en el área de la Educación Física (Klein y Hollingshead, 2015). En los últimos años, distintos estudios han puesto de manifiesto y difundido esa preocupación por la colaboración entre dos o más docentes generalistas (Conderman et al., 2008). No obstante, las evidencias empíricas sobre dicha relación son aún escasas (Rytivaara, 2012).

Entre los componentes esenciales de la co-enseñanza (Scruggs, Mastropieri y McDuffie, 2007), una amplia mayoría de autores coinciden en señalar los siguientes: planificación, instrucción y evaluación (Gately, y Gately, 2001; Ghedin, Aquario y Di Masi, 2013). Respecto a la instrucción, Friend y Cook (2007) proponen una serie de modelos de coenseñanza distinguidos, principalmente, por la responsabilidad o función asumida, la distribución espacial y la organización del alumnado. Por aportar un ejemplo, el nivel más alto (colaborativo) de co-enseñanza es el denominado "enseñanza en equipo" (team teaching), caracterizado por la flexibilidad de roles (p. e., observador) y la reciprocidad. Otros autores (Ploessl, Rock, Schoenfeld y Blanks, 2010) incorporan los componentes de comunicación y resolución de conflictos. En este sentido, Rytivaara (2012) concluye que la docencia compartida se rige por dos tareas fundamentales: la enseñanza y la gestión de clase. Varios "co-docentes" afirman estar acostumbrados a emplear metodologías que no sabrían aplicar de manera individual debido, entre otras razones, al control del grupo-clase (Conderman et al., 2008). Así, Murawski y Dieker (2004) manifiestan la importancia de seleccionar los métodos o estrategias más adecuados a fin de mejorar la intervención.

En el área de la Educación Física (EF), varias metodologías se han mostrado eficaces en el desarrollo social y actitudinal del alumnado, entre las que figuran los modelos de Responsabilidad Personal y Social (Hellison, 2011) o la Educación Deportiva (Siedentop, 1994). El modelo de Educación Deportiva, reconocido como uno de los modelos pedagógicos de la EF (Metzler, 2011), identifica de manera simultánea varios de los elementos anteriormente presentados (p. e., instrucción, evaluación). Este modelo nace con el fin de brindar al alumnado experiencias deportivas auténticas y significativas (Siedentop, Hastie y van der Mars, 2011), asumiendo el reto de crear jugadores competentes, cultos y entusiastas (Siedentop, 1994). El modelo se caracteriza por una serie de rasgos estructurales que emulan al deporte institucionalizado y que han sido debidamente adaptados al contexto educativo (Siedentop et al., 2011): (1) temporadas, que implican la estructura temporal de la 
unidad; (2) afiliación, de modo que el alumnado se organiza mediante equipos; (3) competición formal; (4) evento final, que alude al desenlace de la unidad; (5) registro de datos; y (6) festividad, ya que la temporada transcurre en un ambiente lúdico y de celebración. Las revisiones realizadas hasta la fecha (Araújo, Mesquita y Hastie, 2014; Hastie, Martínez de Ojeda y Calderón, 2011; Wallhead y O’Sullivan, 2005) certifican el potencial del modelo en el desarrollo de variados aprendizajes, ya sea en el plano motor (Mahedero, Calderón, Arias, Hastie y Guarino, 2015), social (Brock, Rovegno y Oliver, 2009) o motivacional (Méndez-Giménez, Martínez de Ojeda y Valverde-Pérez, 2017).

En relación a la población objeto de estudio, las primeras revisiones (Hastie et al., 2011; Wallhead y O'Sullivan, 2005) presentan estudios realizados mayormente en contextos de educación secundaria, siendo más escasas las propuestas emprendidas con alumnado de educación primaria (EP). Si bien en los últimos años los trabajos en este contexto han proliferado, destacando la producción en el ámbito nacional (p, e., Evangelio, González-Víllora, Serra-Olivares y Pastor-Vicedo, 2016), los primeros cursos de EP continúan siendo un escenario escasamente investigado. Así, destacan los estudios de Gutiérrez, García-López, Chaparro y Fernández (2014) y de Layne y Hastie (2016), quienes confirmaron la viabilidad de la Educación Deportiva con alumnado de segundo de EP. Gutiérrez et al. (2014), a partir de una temporada de diana móvil, examinaron los efectos del modelo sobre las metas que persigue (jugadores competentes, cultos y entusiastas), encontrando mejoras en la competencia de juego en equipo. En su caso, la docencia fue asumida por una alumna en prácticas asistida por una maestra colaboradora sin experiencia respecto a la Educación Deportiva. Por su parte, Layne y Hastie (2016) analizaron los efectos del modelo abordando una unidad de habilidades específicas revelando efectos positivos en el entusiasmo y la autonomía del alumnado.

Respecto a la co-enseñanza, son muy pocos los estudios que han analizado su impacto en el contexto de la EF. Por ejemplo, un estudio italiano (Bardaglio, Marasso, Magno, Rabaglietti y Ciairano, 2015), haciendo uso del team teaching, analizó el impacto de tres intervenciones (profesor no especializado, profesor especializado y team-teaching) sobre las habilidades motrices en estudiantes de tercer curso de EP. El modelo de co-enseñanza supuso un plus en el desarrollo de habilidades motrices coordinativas respecto al resto de programas. Más próximos a 
los intereses del estudio actual, Calderón, Martínez de Ojeda, Valverde y Méndez-Giménez (2016) estudiaron el impacto de una intervención basada en la Educación Deportiva sobre el clima social de aula en alumnado de segundo y tercer ciclo de EP, siendo hasta la fecha la única evidencia sobre co-enseñanza y Educación Deportiva. Los autores (Calderón et al., 2016) informaron de mejoras en el comportamiento, la integración y el respeto entre iguales en estudiantes partícipes de grupos "combinados y de docencia compartida".

A pesar de estas valiosas aportaciones, en la actualidad no constan estudios que analicen el efecto de conjugar la Educación Deportiva con estrategias de co-enseñanza en alumnado de primer ciclo de EP (6-8 años). Asimismo, se desconoce el potencial agregado de la co-enseñanza sobre las metas que persigue el modelo.

Por ello, el presente estudio se ha propuesto dos objetivos. Primero, examinar el impacto de una experiencia de co-enseñanza en segundo de EP bajo los postulados de la Educación Deportiva sobre el desarrollo de las metas que persigue el modelo y sobre la percepción discente respecto a sus elementos esenciales. El segundo objetivo se ha centrado en conocer las percepciones de estudiantes y profesores respecto a la intervención realizada. De forma específica, se ha pretendido indagar las posibles diferencias según el género, el país de origen y el grupo-clase de pertenencia. Se establece como hipótesis que la intervención provocará mejoras significativas en cada una de las metas que persigue el modelo, sin existir diferencias intergrupales según las variables referidas.

\section{MÉTODO}

\subsection{Diseño}

El presente estudio se suscribe a un diseño de tipo pre-experimental con medidas pretest y postest. Igualmente, emplea una metodología mixta, valiéndose de instrumentos tanto cuantitativos como cualitativos. Estudios previos con Educación Deportiva han seguido este tipo de diseños (Calderón et al. 2016).

\subsection{Participantes}

La muestra participante estuvo formada por 34 estudiantes (22 chicos y 12 chicas) pertenecientes a dos cursos de segundo de EP de un 
colegio ubicado en el sureste de España. Los estudiantes acudían a clases de EF dos veces por semana. Sus edades oscilaron entre los 7 y 8 años $(M=7.53 ; D T=.50)$, mientras que en cuanto a su procedencia, 11 estudiantes eran autóctonos (españoles) y 23 de origen inmigrante (mayormente marroquíes). Los estudiantes no tenían experiencia previa respecto a la Educación Deportiva. La docencia fue asumida por dos maestros especialistas en EF con desigual experiencia respecto al empleo de la Educación Deportiva (uno experto -4 años aplicándolo- y otro novel), al igual que lo ocurrido en Calderón et al. (2016). El estudio contó con el consentimiento informado tanto del centro educativo como de los padres y madres del alumnado participante.

\subsection{Procedimiento}

El docente experto en Educación Deportiva llevó a cabo el diseño de la intervención. Para ello, contó con la colaboración de su homólogo novel, quien asumió dicha labor como parte de su formación. La validez del modelo fue comprobada mediante el cuestionario de Sinelnikov (2009, traducido al español por Calderón, Hastie y Martínez de Ojeda, 2010). De los ítems propuestos en dicho instrumento, dos no fueron observados en esta unidad: (a) el profesor incorpora la evaluación compartida como parte del proceso de recogida de datos, y (b) los alumnos participan en la selección de los equipos.

Se desarrolló un programa para la enseñanza de un predeporte denominado los cinco pases. Dicho contenido fue seleccionado, entre otras razones, por el requerimiento de habilidades motrices básicas no muy exigentes para estudiantes de esta edad (pases y recepciones), y la inclusión de principios tácticos comunes a deportes de invasión (p.ej., conservación y recuperación del móvil). Las normas que guiaron el juego fueron las siguientes: (a) no está permitido desplazarse con el balón en las manos, (b) no se puede botar el balón, (c) si no se posee el balón en las manos, está permitido moverse por cualquier lugar, (d) si el balón sale fuera, saca el equipo contrario por donde salió, y (e) si se consiguen cinco pases consecutivos se logra un punto, y saca el equipo contrario desde una esquina.

La unidad se desarrolló a lo largo de 12 sesiones de 60 minutos de duración (1 introductoria, 2 dirigidas, 5 de práctica autónoma, 3 de competición formal, y 1 de evento final). La tabla I resume los contenidos de cada sesión en cada fase de la temporada. Siguiendo las 
premisas de la Educación Deportiva, los 34 estudiantes fueron agrupados en ocho equipos (todos formados por cuatro integrantes, salvo dos en los que participaron cinco). Dado que los dos cursos acudían a clases de EF de manera simultánea, los docentes decidieron organizar equipos mixtos en cuanto al grupo-clase de pertenencia, además de seguir criterios de género y país de origen.

Tabla I. Contenidos de las sesiones en cada fase de la temporada de los cinco pases

\begin{tabular}{|c|c|}
\hline $\begin{array}{l}\text { Sesión } \\
\text { (Fase) }\end{array}$ & Contenido \\
\hline 1 (I) & Fichas de equipo, explicación de juego y roles. \\
\hline 2 (D) & $\begin{array}{l}\text { Pases de balón por parejas y en grupo. } \\
\text { Juegos: el mareíllo, pase y paso atrás,... }\end{array}$ \\
\hline 3 (D) & Diferentes tipos de pase (con reflexión sobre su uso), 5 pases 4x0. \\
\hline $4(\mathrm{PR}-\mathrm{AE})$ & Actividades de pase sencillas (con hoja gráfica). 5 pases 3x1. \\
\hline $5(\mathrm{PR}-\mathrm{AE})$ & Actividades de pase sencillas (con hoja gráfica). 5 pases 2x2. \\
\hline $6(\mathrm{PR}-\mathrm{DT})$ & $\begin{array}{l}\text { Actividad autónoma Partido de práctica para aprender a pitar y anotar } \\
\text { cambiando algún elemento para darle componente táctico. }\end{array}$ \\
\hline $7(\mathrm{PR}-\mathrm{DT})$ & $\begin{array}{l}\text { Actividad autónoma Partido de práctica para aprender a pitar y anotar } \\
\text { cambiando algún elemento para darle componente táctico. }\end{array}$ \\
\hline $8(\mathrm{PR}-\mathrm{DT})$ & $\begin{array}{l}\text { Actividad autónoma. Partido de práctica para aprender a pitar y anotar } \\
\text { cambiando algún elemento para darle componente táctico. }\end{array}$ \\
\hline $9(\mathrm{CF})$ & 2 liguillas de 4 equipos. \\
\hline 10 (CF) & 2 liguillas de 4 equipos (continuación). \\
\hline 11 (CF) & $1^{\mathrm{o}} \mathrm{vs} 1^{\mathrm{o}} ; 2^{\mathrm{o}} \mathrm{vs} 2^{\mathrm{o}} ; 3^{\mathrm{o}} \mathrm{vs} 3^{\mathrm{o}} ; 4^{\mathrm{o}} \mathrm{vs} 4^{\mathrm{o}}$ \\
\hline $12(\mathrm{EC})$ & Presentación multimedia y entrega de diplomas \\
\hline
\end{tabular}

Nota: I: Introductoria; D: Dirigida; PR-AE: Práctica - Alumno-Entrenador; PRDT: Práctica - Duty Team; CF: Competición formal; EC: Evento culminante

Las responsabilidades asumidas por el alumnado fueron: (a) entrenador, (b) preparador físico, (c) encargado de material, y (d) responsable de riesgos laborales. En aquellos equipos compuestos por cinco miembros la labor de encargado de material fue compartida. Tanto la formación de los equipos como la selección de los entrenadores fueron realizadas por los docentes, a efectos de garantizar heterogeneidad y 
eficacia en el desarrollo de la unidad. Los roles restantes fueron decididos por los propios estudiantes en sus respectivos equipos. La asignación del rol de entrenador estuvo equilibrada en cuanto a género, mientras que respecto a su lugar de origen, cinco entrenadores fueron inmigrantes y tres españoles. Estos fueron seleccionados atendiendo a distintos aspectos considerados como básicos para el ejercicio del rol (p.ej., liderazgo, responsabilidad y capacidad de comunicación). Siguiendo el carácter festivo del modelo, los equipos adoptaron diferentes elementos distintivos (p.ej. color, escudo, lema o grito inicial).

Como parte de la enseñanza y aprendizaje de los roles, estudiantes de sexto curso de primaria del propio centro asumieron la enseñanza del calentamiento. Respecto a la labor del duty team, una de las tres sesiones dedicadas a su aprendizaje (normas, hoja de anotación, etc.) fue realizada en el aula plumier (sala de TICs, informática...).

\subsection{Instrumentos}

\section{Percepción discente}

Entrevistas. Al finalizar la intervención, los estudiantes fueron entrevistados en grupos de cuatro-cinco personas, siguiendo las recomendaciones de Ennis y Chen (2012). Así, fueron realizadas ocho entrevistas semi-estructuradas, dirigidas por los docentes participantes. Los guiones de las entrevistas fueron supervisados por dos expertos (doctores con, al menos, 10 años de experiencia docente) y abordaron cuestiones relacionadas con el proceso de enseñanza-aprendizaje, así como con los elementos esenciales de la Educación Deportiva. Un ejemplo de pregunta es: "¿Cómo ha sido tu relación con el resto de miembros del equipo?”. Las entrevistas tuvieron una duración aproximada de 30 minutos.

Cuestionario de Educación Deportiva. Se empleó el Physical Education Season Survey (PESS), diseñado por Mohr, Townsend, Rairigh y Mohr (2003), posteriormente ampliado por Hastie y Sinelnikov (2006). La escala consta de 12 preguntas distribuidas en dos secciones: en primer lugar (preguntas 1-6), el alumnado hace una valoración de cada uno de los seis elementos esenciales de la Educación Deportiva (temporada, afiliación, registro de datos, competición formal, festividad y evento final), justificando su elección mediante una breve respuesta cualitativa. En segundo lugar (preguntas 7-12), los participantes hacen una autovaloración de cada uno de los tres objetivos perseguido por el 
modelo en relación a antes y después de haber participado en la unidad (competencia, "puntúa sobre tus habilidades en los cinco pases a lo largo de la temporada"; cultura deportiva, "puntúa sobre cuánto crees que sabes sobre los cinco pases"; y entusiasmo, "indica cuánto te gusta la Educación Física antes/después de participar en la temporada de cinco pases").

La primera sección fue valorada en una escala de 1 a 5 mientras que la segunda sección emplea una escala de 1 a 10, suponiendo en cada caso el valor mínimo "totalmente en desacuerdo" y el máximo "totalmente de acuerdo". Los cuestionarios fueron administrados en una sesión de contingencia, en el aula de referencia de cada grupo-clase, invirtiendo un tiempo aproximado de 25 minutos para su realización.

\section{Percepción docente}

Entrevistas. Cada docente fue sometido a tres entrevistas semiestructuradas (Cohen y Manion, 2002) a efectos de conocer su opinión en diferentes momentos de la intervención (antes, durante y después de la unidad). Las entrevistas tuvieron una duración aproximada de 20 minutos cada una. Un ejemplo de pregunta es: “¿Qué aspectos propios de la Educación Deportiva se han visto potenciados al ser abordados mediante docencia compartida?”.

Diarios. Asimismo, los maestros participantes dieron cuenta de sus observaciones e impresiones más significativas a través de un diario (Erickson, 1989) en el que se abordaron cuestiones previamente consensuadas (p. e., objetivos del modelo) y otras de gran relevancia que pudiesen emerger (p. e., comportamientos autónomos).

\section{5. Análisis de datos}

Los datos recabados a través del cuestionario cuantitativo fueron analizados usando el paquete estadístico IBM-SPSS (versión 23.0). El cociente alfa de Cronbach determinó la consistencia interna de las variables analizadas. Siguiendo lo realizado por Burgueño, Lirola, Morales-Ortíz, Cueto-Martín y Medina-Casaubón (2018), los seis elementos nucleares de la Educación Deportiva fueron considerados de forma unidimensional, mostrando niveles de $\alpha>$.68. Estos valores no alcanzaron los niveles recomendados ( $\alpha>$.70; Kline, 1998), sin embargo fueron mantenidos para los análisis subsiguientes debido a la franja de edad del alumnado y el interés que suponía para el estudio. Por su parte, 
la fiabilidad de las variables de la segunda sección del PESS no fue analizada dado que se trató de escalas de un solo ítem. Se calcularon los descriptivos (medias y desviaciones típicas) y la inferencia estadística mediante la prueba de rangos de Wilcoxon, con el fin de contrastar los efectos de la intervención. Igualmente, se efectuó la prueba $U$ de MannWhitney a efectos de analizar comparaciones intergrupo (género, país de origen y grupo-clase). En cada caso, el nivel de significación estadística se estableció en $p<.05$.

Los datos cualitativos fueron codificados y reagrupados a partir de una serie de temas emergentes (p. e., diversión). Una vez codificadas, todas las respuestas fueron trasladadas a una matriz de frecuencia a efectos de valorar su frecuencia relativa y su grado de relevancia respecto al tópico de pertenencia. Una tabulación posterior sugirió conexiones significativas entre varios tópicos, resultando en las categorías presentes en este estudio: (a) planificación didáctica. Consideraciones respecto a los aspectos que definen la ED, y (b) asunción de roles: implicaciones respecto a aquellos de mayor centralidad.

\section{RESUltados}

\section{1. Resultados cuantitativos}

A continuación, la tabla II recoge las puntuaciones dadas por el alumnado en relación a los elementos esenciales de la Educación Deportiva, desglosadas según condición grupal. En líneas generales, se observa que todas las puntuaciones estuvieron por encima de 4, con la única excepción de la afiliación en el alumnado autóctono $(M=3.91)$. Los valores más altos se dieron en los aspectos de temporada y registro, siendo así para alumnado de sexo masculino, así como aquel de origen inmigrante. El alumnado autóctono dio igual prioridad a los aspectos de registro, estructura y festividad. En relación al género, las mujeres dieron mayor importancia a la temporada y a la festividad. Por su parte, los valores más bajos emergieron en la afiliación. Considerando el grupoclase de pertenencia, el curso con docente experto valoró más el registro, mientras que el curso con docente novel enfatizó la noción de temporada. 
Tabla II. Estadísticos descriptivos de las percepciones de los estudiantes sobre los elementos del MED para la muestra total, género, país de origen y grupo-clase de pertenencia

\begin{tabular}{|c|c|c|c|c|c|c|c|c|c|c|c|c|}
\hline & \multicolumn{2}{|c|}{ Temporada } & \multicolumn{2}{|c|}{ Afiliación } & \multicolumn{2}{|c|}{$\begin{array}{l}\text { Registro de } \\
\text { datos }\end{array}$} & \multicolumn{2}{|c|}{$\begin{array}{l}\text { Competició } \\
\text { n formal }\end{array}$} & \multicolumn{2}{|c|}{ Festividad } & \multicolumn{2}{|c|}{ Evento final } \\
\hline & $M$ & $D T$ & $M$ & $D T$ & $M$ & $D T$ & $M$ & $D T$ & $M$ & $D T$ & $M$ & $D T$ \\
\hline N Total & 4.88 & .40 & 4.26 & 1.13 & 4.85 & .35 & 4.74 & .44 & 4.78 & .79 & 4.68 & .63 \\
\hline Varones & 4.86 & .46 & 4.18 & 1.25 & 4.86 & .35 & 4.68 & .47 & 4.73 & .93 & 4.59 & .73 \\
\hline Mujeres & 4.92 & .28 & 4.42 & .90 & 4.83 & .38 & 4.83 & .38 & 4.90 & .31 & 4.83 & .38 \\
\hline Autóctonos & 4.73 & .64 & 3.91 & 1.22 & 4.82 & .40 & 4.82 & .40 & 4.82 & .60 & 4.64 & .67 \\
\hline Inmigrantes & 4.96 & .20 & 4.43 & 1.08 & 4.87 & .34 & 4.70 & .47 & 4.76 & .88 & 4.70 & .63 \\
\hline D. Experto & 4.89 & .45 & 4.16 & 1.16 & 4.95 & .22 & 4.89 & .31 & 4.89 & .47 & 4.68 & .58 \\
\hline D. Novel & 4.87 & .35 & 4.40 & 1.12 & 4.73 & .45 & 4.53 & .51 & 4.64 & 1.08 & 4.67 & .72 \\
\hline
\end{tabular}

Nota: $M=$ Media; $D T$ = Desviación típica

Con respecto a los objetivos que persigue el modelo, se hallaron aumentos significativos en cada una de las variables y condiciones grupales (ver tabla III). Los cambios de mayor significatividad se sucedieron en la competencia y cultura deportiva, principalmente en el alumnado inmigrante y en ambos cursos y géneros. En relación al entusiasmo, se observaron aumentos significativos en la muestra total, así como en el alumnado de sexo femenino, inmigrante y perteneciente al curso con docente novel.

(Tabla III, página siguiente) 
Tabla III. Estadísticos descriptivos de la percepción de los estudiantes sobre su competencia, cultura deportiva y entusiasmo por la EF de la muestra total, género, procedencia y grupo-clase

\begin{tabular}{|c|c|c|c|c|c|c|}
\hline & \multicolumn{2}{|c|}{ Competencia } & \multicolumn{2}{|c|}{ Cultura deportiva } & \multicolumn{2}{|c|}{ Entusiasmo } \\
\hline & Pre & Post & Pre & Post & Pre & Post \\
\hline N Total & $\begin{array}{c}5.45 \\
(2.46)\end{array}$ & $\begin{array}{c}9.12 * * * \\
(1.05)\end{array}$ & $\begin{array}{c}5.64 \\
(2.82)\end{array}$ & $\begin{array}{c}9.15^{* * *} \\
(1.46)\end{array}$ & $\begin{array}{c}7.75 \\
(2.82)\end{array}$ & $\begin{array}{l}9.39 * \\
(1.39)\end{array}$ \\
\hline Mujeres & $\begin{array}{c}5.83 \\
(1.52)\end{array}$ & $\begin{array}{c}9.33^{* *} \\
(.65)\end{array}$ & $\begin{array}{c}5.50 \\
(2.36)\end{array}$ & $\begin{array}{c}9.33^{* *} \\
(1.60)\end{array}$ & $\begin{array}{c}6.58 \\
(3.32)\end{array}$ & $\begin{array}{l}9.67 * \\
(1.61)\end{array}$ \\
\hline Varones & $\begin{array}{c}5.24 \\
(2.88)\end{array}$ & $\begin{array}{c}9.00 * * \\
(1.23)\end{array}$ & $\begin{array}{c}5.71 \\
(3.12)\end{array}$ & $\begin{array}{c}9.05^{* *} \\
(1.60)\end{array}$ & $\begin{array}{c}8.45 \\
(2.31)\end{array}$ & $\begin{array}{c}9.24 \\
(1.61)\end{array}$ \\
\hline Autóctonos & $\begin{array}{c}6.00 \\
(2.72)\end{array}$ & $\begin{array}{c}9.36 * \\
(.92)\end{array}$ & $\begin{array}{c}5.36 \\
(3.11)\end{array}$ & $\begin{array}{l}9.36 * \\
(1.80)\end{array}$ & $\begin{array}{c}8.80 \\
(1.32)\end{array}$ & $\begin{array}{c}9.45 \\
(1.21)\end{array}$ \\
\hline Inmigrantes & $\begin{array}{c}5.18 \\
(2.34)\end{array}$ & $\begin{array}{c}9.00 * * * \\
(1.11)\end{array}$ & $\begin{array}{c}5.77 \\
(2.74)\end{array}$ & $\begin{array}{c}9.05 * * * \\
(1.29)\end{array}$ & $\begin{array}{c}7.27 \\
(3.21)\end{array}$ & $\begin{array}{l}9.36 * \\
(1.50)\end{array}$ \\
\hline D. Experto & $\begin{array}{c}6.33 \\
(2.40)\end{array}$ & $\begin{array}{c}9.22 * * \\
(.81)\end{array}$ & $\begin{array}{c}6.17 \\
(3.07)\end{array}$ & $\begin{array}{c}9.00 * * \\
(1.53)\end{array}$ & $\begin{array}{c}8.22 \\
(2.76)\end{array}$ & $\begin{array}{c}9.33 \\
(1.28)\end{array}$ \\
\hline D. Novel & $\begin{array}{c}4.40 \\
(2.17)\end{array}$ & $\begin{array}{c}9.00 * * \\
(1.31)\end{array}$ & $\begin{array}{c}5.00 \\
(2.45)\end{array}$ & $\begin{array}{c}7.14^{* *} \\
(1.40)\end{array}$ & $\begin{array}{c}9.33 \\
(2.91)\end{array}$ & $\begin{array}{l}9.47^{*} \\
(1.55)\end{array}$ \\
\hline
\end{tabular}

Nota: Desviación típica entre paréntesis

Finalmente, las comparaciones intergrupo efectuadas a partir de la prueba $U$ de Mann-Whitney revelaron diferencias estadísticamente significativas en la competición formal según el grupo de pertenencia ( $U$ de Mann-Whitney = 91.000; $Z=-2.337 ; p=.019$ ), sin encontrar diferencias en relación al género y al país de origen. Por su parte, no se hallaron diferencias significativas en ninguna de las metas establecidas por el modelo.

\section{2. Resultados cualitativos}

A continuación, se presenta la información cualitativa considerando las dos categorías extraídas del análisis de los diarios, cuestionarios y entrevistas tanto al profesorado como al alumnado. 


\section{Planificación didáctica. Consideraciones respecto a los aspectos que definen la ED}

Previo al inicio de la unidad, los docentes afrontaron de forma análoga el proceso de planificación. El maestro novel aseguró ser muy similar al realizado en cursos más avanzados, anticipando que los conflictos surgirían durante la puesta en práctica de la intervención. El docente experto afirmó que las decisiones preactivas estarían condicionadas por la edad del alumnado. Entendió que algunas fases, una vez iniciadas, podrían requerir ajustes temporales. Respecto a las actividades, argumentó que deberían ser más sencillas, a efectos de garantizar mayores oportunidades de éxito. Cabe destacar que, en muchas de las decisiones docentes, la madurez del alumnado emergió como constante explicativa. Fue el caso de la afiliación, sobre la que el docente experto explicó: "La elección de equipos la hemos realizado los profesores ya que, si están bien realizadas las elecciones, está más asegurado el éxito, máxime para una edad temprana”. Añadiendo que podría afectar a las relaciones sociales de los estudiantes, al verse obligados a trabajar con personas con las que no acostumbraban. Su homólogo novel, en consonancia con lo anterior, explicó: "A la hora de trabajar como grupo, las relaciones sociales les cuestan un poco más. Su capacidad de autocontrol está poco desarrollada”. Por su parte, la opinión discente fue de conformidad respecto a los agrupamientos y la buena calidad de las relaciones.

Sin embargo, los maestros observaron conflictos puntuales devenidos, principalmente, por la propia dinámica grupal: "Surge alguna pequeña discusión cuando deben tomar decisiones de forma autónoma”, observó el docente experto. En este sentido, algunos estudiantes manifestaron haber percibido comportamientos negativos por parte de sus iguales: "Cuando nos salía algo mal, el entrenador nos 'gruñía' mucho". No obstante, estas disputas generalmente no requerían de intervención docente ya que el desarrollo de una mayor conciencia grupal contribuía a resolverlas. En este sentido, numerosos estudiantes destacaron las bondades de elementos festivos como el grito, el color o lema de equipo, mientras que los docentes dieron mayor importancia al nombre o color: "Ellos oyen el nombre de su equipo y enseguida se arman. Se disponen rápidamente”, dijo el docente experto.

Los maestros dieron cuenta de los efectos generados por las fases sobre el aprendizaje de los estudiantes. Anticiparon con acierto que las mayores dificultades se presentarían en las primeras sesiones de la 
unidad: "Será complicado porque van a estar pendientes de muchas cosas" (docente experto). Una de las dificultadas reportadas por el profesorado estribó en los períodos de transición: "Cada vez que se inicia una nueva fase se genera cierto desconcierto", comentó el maestro experto. Como solución, apuntó la conveniencia de establecer ritmos más lentos que llevasen aparejados un recordatorio de los objetivos perseguidos y las responsabilidades asumidas. La fase de competición formal fue reconocida por el profesorado como motivante.

El alumnado valoró positivamente la estructura formal de la temporada, percibiendo la alternancia de partidos de práctica y de competición como oportunidad de preparación. Igualmente, desde la perspectiva docente, se destacó el nivel de autonomía logrado por el alumnado durante su participación en las fases finales de la temporada: "No hemos intervenido para nada. Solo hemos estado observando cómo han competido, pitado, etc. En ese sentido, ha salido bastante bien”, dijo el maestro experto. En consonancia con ello, su colega novel añadió: "Con el paso de las sesiones los problemas se van disipando y nos sorprende observar la capacidad de adaptación de los niños para cada una de las fases". Respecto al evento culminante, tanto alumnado como profesorado destacaron efectos positivos sobre el entusiasmo: " $\mathrm{Va} a$ tener éxito la unidad y sus partes. Concretamente, el evento final como colofón" (docente novel); "Es algo afectivo. Los niños se entusiasman" (docente experto). Al finalizar la experiencia, los estudiantes valoraron, casi de forma unánime, la entrega de diplomas como el momento de mayor satisfacción.

\section{Asunción de roles: implicaciones respecto a aquellos de mayor centralidad}

Las expectativas docentes fueron alentadoras respecto al desempeño de roles más "operativos" (preparador físico, encargado del material, etc.), si bien, en el transcurso de la unidad, el docente experto observó ligeros contratiempos: "Hubo un poco de caos. Tuvimos que volver a recordar los roles y estar muy pendientes en cada momento". Las dificultades se fueron disipando conforme avanzaron las sesiones, tal como fue constatado por ambos maestros: "Los roles se están empezando a cumplir de forma efectiva" (docente novel); "Los roles funcionan bien” (docente experto). Finalizada la temporada, el maestro novel apuntó mejoras de gran relevancia: "Cada uno sabe cuál es su rol y lo sabe desarrollar a la perfección. Te diré más, saben desarrollar su rol y 
el de los demás también". Sin embargo, según los comentarios docentes, los roles de mayor centralidad (p. e., alumno entrenador) delinearon un panorama mucho más peliagudo, emergiendo varias apreciaciones a ser tenidas en cuenta para su abordaje con alumnado de segundo de EP.

a) Alumno entrenador. El ejercicio del rol de alumno entrenador fue percibido por los docentes como un reto sustantivo. Exponiendo razones de madurez, los maestros abogaron por seleccionar a aquellos estudiantes más aptos para el rol. Las primeras sensaciones fueron positivas, destacando la capacidad de los entrenadores para transmitir correctamente las actividades propuestas, así como para gestionar el grupo: "Cada vez ha ido mejor y, hacia el final de la sesión, hemos comprobado cómo los entrenadores han sido capaces de controlar las situaciones" (docente experto). Otras consideraciones didácticas incluyeron la importancia de partir de actividades previamente conocidas por el alumnado. Las dificultades más significativas sucedieron al introducir nuevas actividades que acarreaban ligeros cambios en su estructura (p. e., número de jugadores). Asimismo, algunos estudiantes, que no ejercían como entrenadores, invadían las responsabilidades de éstos en un intento por obtener mayor protagonismo.

b) Duty team. Antes de comenzar la temporada, el profesorado se mostró muy escéptico respecto al desarrollo de la labor arbitral: "No va a acabar funcionando bien. Les va a costar mucho" (maestro experto). Sin embargo, los docentes percibieron ligeras mejoras tras la primera sesión específica de duty team. A nivel específico, observaron cómo el arbitraje, a diferencia de la anotación, requería mayor apoyo docente. En este sentido, los docentes advirtieron la dificultad de aplicar reglas en contextos de juego real en estas edades. Un hecho que contribuyó al desarrollo de este rol fue la sesión teórica dedicada a explicar las funciones del duty team: "Creo que esa sesión intermedia ha sido muy fructífera”, declaró el maestro experto. Los estudiantes mostraron mayor predilección por la anotación, si bien no expresaron disgusto por el arbitraje. Los anotadores, si bien desarrollaron su labor con facilidad, solían completar la hoja de anotación antes de que finalizara el partido, siendo un error frecuente, según el profesorado.

\section{DisCUSIÓN}

El estudio actual se ha propuesto examinar el impacto de una experiencia de co-enseñanza en segundo curso de EP bajo los postulados 
de la Educación Deportiva sobre el desarrollo de las metas que persigue el modelo y sobre la percepción discente respecto a sus elementos esenciales. Un segundo objetivo se ha centrado en conocer las percepciones de estudiantes y profesores respecto a la intervención realizada. En general, los resultados del presente estudio dan soporte a los hallazgos previos sobre las bondades de conjugar estrategias de coenseñanza con la Educación Deportiva (Calderón et al., 2016), haciendo extensibles sus beneficios a cursos iniciales de EP. Asimismo, complementan evidencias respecto al potencial del modelo para ser introducido en alumnado de corta edad (Gutiérrez et al., 2014; Layne y Hastie, 2016).

Respecto a los objetivos propuestos, fueron revelados aumentos significativos en las tres metas perseguidas por el modelo, confirmando así la hipótesis establecida. Además, en concordancia con esta última, no se revelaron diferencias intergrupales por razón del género, el país de origen o el grupo-clase de pertenencia. Las mejoras se presentaron en cada una de las condiciones grupales, a excepción del entusiasmo en varones, alumnado autóctono y estudiantes del grupo con docente experto. Diversos aspectos didácticos parecieron explicar mejoras competenciales. Primero, se confirmaron los beneficios que supone partir de actividades previamente conocidas, con sencillos requerimientos, yendo en línea de lo advertido por Gutiérrez et al. (2014) y Layne y Hastie (2016) quienes abogaron por una elección precisa del contenido.

Asimismo, se apuntó la necesidad de establecer ritmos lentos durante las primeras sesiones, prestando especial atención a la transición de una fase a otra (p.ej., dirigida hacia práctica autónoma). Los períodos de transición parecen mostrarse sensibles a las diferencias individuales (Puente-Maxera, Méndez-Giménez y Martínez de Ojeda, 2018). En términos de co-enseñanza, la observación general sobre estudiantes con distintas velocidades, así como los intentos por recabar una valoración integral del aprendizaje, se lograría más eficientemente con más de un docente (Murawski y Dieker, 2004). En este sentido, la estructura formal de la temporada, con su alternancia entre partidos amistosos y de competición, fue percibida por el alumnado como oportunidad de aprendizaje y de preparación para afrontar la competición en las mejores condiciones (Gutiérrez, García-López, Hastie y Calderón, 2013). La participación en partidos ha sido revelada como promotora de una mayor percepción de competencia (Perlman y Goc Karp, 2010). 
Igualmente, el objetivo de cultura deportiva experimentó aumentos en todas las condiciones grupales, siendo congruente con intervenciones previas en EP (Calderón et al., 2010; Gutiérrez et al., 2014). Desde el plano cualitativo, fue reseñable el conocimiento adquirido respecto al funcionamiento de distintos roles, además del propiamente asignado (García-López, Gutiérrez, González-Víllora y Valero, 2012; Hastie y Sinelnikov, 2006). Las dificultades advertidas en aquellos de mayor centralidad exhortan sobre la necesaria atención en cursos iniciales de EP. Estudios previos ya han puesto de manifiesto la complejidad de la labor arbitral (Calderón, et al. 2010). Respecto al alumno entrenador, los datos cualitativos informaron de inconvenientes (p.ej., cambios en la estructura de la actividad), lo que sugiere la inclusión de protocolos de formación específica en futuras intervenciones (Farias, Hastie y Mesquita, 2017).

En tercer lugar, la intervención provocó mejoras en el entusiasmo de los participantes, existiendo diferencias parciales al desglosar por género (mujeres), procedencia (inmigrantes) y grupo-clase de pertenencia (docente novel). Estudios previos en primer y segundo ciclo de EP han revelado aumentos en el entusiasmo de los escolares (Calderón et al. 2010; Layne y Hastie, 2016). Entre los aspectos que dieron cuenta de una participación entusiasta, los datos cualitativos ponen de manifiesto el efecto positivo de determinadas fases específicas de la temporada. Las fases han sido asiduamente refrendadas como condición ambiental positiva de la Educación Deportiva (Sinelnikov y Hastie, 2010). Así, la competición formal fue revelada como motivante, destacando la expectativa discente generada hacia los enfrentamientos con sus iguales (MacPhail, Gorely, Kirk y Kinchin, 2008). Del mismo modo, el evento final favoreció un aumento en la excitación del alumnado (Calderón et al. 2010; Hastie y Sinelnikov, 2006; Layne y Hastie, 2016) siendo declarado como el momento de mayor satisfacción de la temporada. Fue evidente en las voces de muchos estudiantes el entusiasmo derivado de enfrentarse a personas de otros cursos, apuntando en la dirección de los hallazgos de Kinchin, MacPhail y Ni Chroinin (2009) respecto a un evento final interescolar.

\section{CONCLUSIONES}

Los resultados del presente estudio dan soporte a hallazgos previos sobre las bondades de combinar la co-enseñanza con el modelo de 
Educación Deportiva (Calderón et al., 2016). Igualmente, apuntan la conveniencia de introducir la Educación Deportiva en estadios iniciales de la EP, dada su incidencia positiva en vistas a futuras versiones del modelo. No obstante, es obligado mencionar que el estudio presenta varias limitaciones. En primer lugar, el propio diseño de investigación, carente de grupo control (a diferencia de lo realizado por Calderón et al., 2016), hace difícilmente la generalización de resultados. Asimismo, el tamaño de la muestra y la concreción del estudio a un único centro y en una única etapa educativa implican limitaciones de importancia, sugiriendo la exploración de nuevos escenarios (p. e., alumnado de educación secundaria). La experiencia docente y discente respecto al modelo supone otro aspecto a considerar en próximas experiencias. Finalmente, futuras intervenciones podrán contemplar el efecto provocado de combinar la co-enseñanza con otros modelos pedagógicos (p. e., modelo comprensivo).

\section{BiBLIOGRAFÍA}

Araújo, R., Mesquita, I. y Hastie, P. A. (2014). Review of the Status of Learning in Research on Sport Education: Future Research and Practice. Journal of Sports Science and Medicine, 13, 846-858.

Bardaglio, G., Marasso, D., Magno, F., Rabaglietti, E. y Ciairano, S. (2015). Team-teaching in physical education for promoting coordinative motor skills in children: the more you invest the more you get. Physical Education and Sport Pedagogy, 20(3), 268-282. doi: 10.1080/17408989.2013.837434

Brock, S. J., Rovegno, I. y Oliver, K. L. (2009). The influence of student status on student interactions and experiences during a sport education unit. Physical Education and Sport Pedagogy, 14(4), 355-375. doi:10.1080/17408980802400494

Burgueño, R., Lirola, M-J., Morales-Ortiz, E., Cueto-Martín, B., y MedinaCasaubón, J. (2018). Análisis psicométrico de la Physical Education Season Survey en estudiantes españoles de educación secundaria. Psychology, Society, \& Education, 10(2), 189-201. doi: 10.25115/psye.v10i1.1093 
Calderón, A., Hastie, P. A. y Martínez de Ojeda, D. (2010). Aprendiendo a enseñar mediante el modelo de Educación Deportiva. Experiencia inicial en Educación Primaria. Cultura, Ciencia y Deporte, 5, 169-180. doi:10.12800/ccd.v5i15.103

Calderón, A., Martínez de Ojeda, D., Valverde, J. J. y Méndez-Giménez, A. (2016). "Ahora nos ayudamos más": Docencia compartida y clima social de aula. Experiencia con el modelo de Educación Deportiva. RICYDE. Revista internacional de ciencias del deporte, 44(12), 121-136. doi: 10.5232/ricyde 2016.04403

Cohen, L. y Manion, L. (2002). Métodos de investigación educativa. Madrid: La Muralla.

Conderman, G., Bresnahan, V. y Pedersen, T. (2008). Purposeful co-teaching: Real cases and effective strategies. Thousand Oaks, CA: Corwin Press.

Ennis, C. D. y Chen, S. (2012). Interviews and focus groups. En K. Armour, D. Macdonald, (Ed.), Research Methods in Physical Education and Youth Sport (pp. 217-236). New York: Routledge.

Erickson, F. (1989). Métodos Cualitativos de Investigación Sobre la Enseñanza. En M. Wittrock, (Ed.). La Investigación de la Enseñanza, II - Métodos Cualitativos y de Observación (pp. 203-47). Barcelona: Paidós-MEc.

Evangelio, C., González-Víllora, S., Serra-Olivares, J. y Pastor-Vicedo, J. C. (2016). El Modelo de Educación Deportiva en España: Una revisión del estado de la cuestión y prospectiva. Cuadernos de Psicología Del Deporte, 16(1), 307-324.

Farias, C., Hastie, P. A. y Mesquita, I. (2018). Scaffolding student-coaches' instructional leadership toward student-centred peer interactions: A yearlong action-research intervention in sport education. European Physical Education Review, 24(3), 269-291.

doi: 10.1177/1356336X16687303

Friend, M. y Cook, L. (2007). Interactions: Collaboration skills for school professionals ( $5^{\mathrm{a}}$ ed.). Boston, MA: Allyn and Bacon.

Gately, S. y Gately, F. (2001). Understanding co-teaching components. Teaching Exceptional Children, 33, 40-47. 
García-López, L. M., Gutiérrez, D., González-Víllora, S. y Valero, A. (2012). Cambios en la empatía, la asertividad y las relaciones sociales por la aplicación del modelo de instrucción educación deportiva. Revista de Psicología del Deporte, 21(2), 321-330.

Ghedin, E., Aquario, D. y Di Masi, D. (2013). Co-teaching in action. Una proposta per promuovere l'educazione inclusiva. Giornale Italiano della Ricerca Didattica, 11, 157-175.

Gutiérrez, D., García-López, L. M., Chaparro, R. y Fernández, A. J. (2014). Aplicación del modelo de Educación Deportiva en segundo de Educación Primaria: percepciones del alumnado y el profesorado. Cuadernos de Psicología del Deporte, 14(2), 131-144.

Gutiérrez, D., García-López, L. M., Hastie, P. A. y Calderón, A. (2013). Spanish students' perceptions of their participation in seasons of sport education. The Global Journal of Health and Physical Education Pedagogy, 2(2), 111-127.

Hastie, P. A., Martínez de Ojeda, D. y Calderón, A. (2011). A review of research on Sport Education: 2004 to the present. Physical Education and Sport Pedagogy, 16(2), 103-132. doi:10.1080/17408989.2010.535202

Hastie, P. A. y Sinelnikov, O. A. (2006). Russian students' participation in and perceptions of a season of Sport Education. European Physical Education Review, 12(2), 131-150. doi:10.1177/1356336X06065166

Hellison, D. (2011). Teaching Personal and Social Responsibility through physical activity ( $3^{\mathrm{a}}$ ed.). Champaign, IL: Human Kinetics.

Keefe, E. B. y Moore, V. (2004). The challenge of co-teaching in inclusive classrooms at the high school level: What the teachers told us. American Secondary Education, 32(3), 77-88. Obtenido el 15 de octubre, 2018 de: https://www.jstor.org/stable/pdf/41064524.pdf

Klein, E. y Hollingshead, A. (2015). Collaboration between special and physical education: The benefits of a healthy lifestyle for all students. Teaching Exceptional Children, 47(3), 163-171. doi: 10.1177/0040059914558945

Kline, P. (1998). The new psychometrics: Science, psychology and measurement. Nueva York: Routledge. 
Kinchin, G. D., MacPhail, A. y Ni Chroinin, D. (2009). Pupils and teachers perceptions of a culminating festival within a Sport Education season in Irish primary schools. Physical Education and Sport Pedagogy, 14(4), 391406. doi: $10.1080 / 17408980802584982$

Layne, T. y Hastie, P. A. (2016). Analysis of teaching physical education to second-grade students using sport education. Education 3-13, 44(2), 226240. doi: 10.1080/03004279.2014.914551

Mahedero, M. P., Calderón, A., Arias, J. L., Hastie, P. A. y Guarino, A. (2015). Effects of student skill level on knowledge, decision making, skill execution and game performance in a mini-volleyball sport education season. Journal of Teaching in Physical Education, 34, 626-641. doi:10.1123/jtpe.2014-0061

MacPhail, A., Gorely, T., Kirk, D. y Kinchin, G. D. (2008). Children's experiences of fun and enjoyment during a season of Sport Education. Research Quarterly for Exercise and Sport, 79(3), 344-355. doi: 10.1080/02701367.2008.10599498

Méndez-Giménez, A., Martínez de Ojeda, D. y Valverde-Pérez, J. J. (2017). Inteligencia emocional y mediadores motivacionales en una temporada de Educación Deportiva sobre mimo. Ágora para la Educación Física y el Deporte, 19(1), 52-72. doi: 10.24197/aefd.1.2017.52-72

Metzler, M. W. (2011). Instructional models for physical education ( $2^{\mathrm{a}}$ ed.). Scottsdale. AZ: Holcomb Hathaway Publishing.

Mohr, D. J., Townsend, J. S., Rairigh, R. y Mohr, C. (2003). Students' perceptions of Sport Education when taught using the pedagogical approach to Sport Education (PASE) planning and instructional framework. Research Quarterly for Exercise and Sport, 74, A-51.

Murawski, W. y Dieker, L. (2004). Tips and strategies for co-teaching at the secondary level. Teaching Exceptional Children, 36(5), 52-58. doi: $10.1177 / 004005990403600507$

Perlman, D. J. y Goc Karp, G. (2010). A self-determined perspective of the sport education model. Physical Education and Sport Pedagogy, 15, 401418. doi:10.1080/17408980903535800 
Puente-Maxera, F., Méndez-Giménez, A. y Martínez de Ojeda, D. (2018). Modelo de Educación Deportiva y dinámica de roles. Efectos de una intervención sobre las variables motivacionales de estudiantes de primaria. Cultura, Ciencia y Deporte, 39(13), 281-290. doi: 10.12800/ccd.v1i1.1149

Ploessl, D. M., Rock, M. L., Schoenfeld, N. y Blanks, B. (2010). Practical techniques to enhance co-teaching interactions. Intervention in School and Clinic, 45(3), 158-168. Obtenido el 3 de noviembre de 2010 de: https://pdfs.semanticscholar.org/94e0/e391e81a0cc3ac22e8a825b73c2e6dc 6d53a.pdf

Santos, M. A. (2000). El pensamiento complejo y la pedagogía. Bases para una teoría holística de la educación. Estudios pedagógicos, 26, 133-148. doi: 10.4067/S0718-07052000000100011

Rytivaara, A. (2012). Collaborative classroom management in a co-taught primary school classroom. International Journal of Educational Research, 53(1), 182-191. doi:10.1016/j.ijer.2012.03.008

Scruggs, T. E., Mastropieri, M. A. y McDuffie, K. A. (2007). Co-teaching in inclusive classrooms: A metasynthesis of qualitative research. Exceptional Children, 73, 392-416. doi: 10.1177/001440290707300401

Siedentop, D. (1994). Sport education: Quality PE through positive sport experiences. Champaign, IL: Human Kinetics.

Siedentop, D., Hastie, P. A. \& van der Mars, H. (2011). Complete Guide to Sport Education ( $2^{\mathrm{a}}$ ed.). Champaign, IL: Human Kinetics.

Sinelnikov, O. A. (2009). Sport education for teachers: Professional development when introducing a novel curriculum model. European Physical Education Review, 15, 91- 114. doi: 10.1177/1356336X09105213

Sinelnikov, O. A. y Hastie, P. A. (2010). A motivational analysis of a season of Sport Education. Physical Education and Sport Pedagogy, 15(1), 55-69. doi: 10.1080/17408980902729362

Wallhead, T. L. y O’Sullivan, M. (2005). Sport education: Physical education for the new millennium? Physical Education and Sport Pedagogy, 10(2), 181-210. doi: 10.1080/17408980500105098 